\title{
The Value of Three-Dimensional Tomographic Ultrasound Imaging under Backpropagation Algorithm in the Local Staging Diagnosis of Cervical Cancer
}

\author{
Chenjie Li $\mathbb{D}^{1},{ }^{1}$ Ziwen Zhang $\mathbb{D}^{2},{ }^{2}$ Yingying Tian $\mathbb{D}^{3},{ }^{3}$ and Bingjie Liu $\mathbb{D D}^{3}$ \\ ${ }^{1}$ Department of Ultrasound, The Affiliated Suzhou Hospital of Nanjing Medical University, Suzhou 215000, Jiangsu, China \\ ${ }^{2}$ Department of Ultrasound, Linping Campus, The Second Affiliated Hospital of Zhejiang University School of Medicine, \\ Hangzhou 311100, China \\ ${ }^{3}$ Department of Ultrasound, The Affiliated Suzhou Science \& Technology Town Hospital of Nanjing Medical University, \\ Suzhou 215000, Jiangsu, China \\ Correspondence should be addressed to Bingjie Liu; 1923131215@siit.edu.cn
}

Received 23 November 2021; Revised 17 January 2022; Accepted 20 January 2022; Published 28 February 2022

Academic Editor: M Pallikonda Rajasekaran

Copyright (c) 2022 Chenjie Li et al. This is an open access article distributed under the Creative Commons Attribution License, which permits unrestricted use, distribution, and reproduction in any medium, provided the original work is properly cited.

\begin{abstract}
Based on three-dimensional tomographic ultrasound imaging (TUI) of the backpropagation algorithm, this study evaluated the consistency of three-dimensional TUI technology, pelvic examination, and magnetic resonance imaging (MRI) technology in the local staging diagnosis of cervical cancer, aiming to explore the application value of TUI technology in local staging diagnosis of cervical cancer. The backpropagation algorithm was adopted to segment images, and 35 patients who were confirmed to be cervical cancer by tissue biopsy were included in this study. All selected patients underwent transvaginal TUI examination and pelvic MRI examination. Then, the 2010 Federation Internationale of Gynecologie and Obstetrigue (FIGO) was adopted to determine the clinical stage, and the $\mathrm{K}$ index and the percentage of consistency were calculated to evaluate the consistency between TUI and MRI. Comparison of tumor size measurements showed that obvious correlation could be found between MRI and threedimensional TUI $(r=0.842 ; P<0.001)$, MRI, and clinical examination $(r=0.654 ; P<0.001)$ to the assessment of tumor size. In terms of parauterine invasion, there was a moderate consistency between pelvic examination and MRI $(K=0.413$; $95 \%$ confidential interval $(\mathrm{CI})=0.085-0.762$; the overall coincidence rate $(\mathrm{OCR})=78.0 \%)$, and there was good consistency between threedimensional TUI and MRI $(K=0.655,95 \% \mathrm{CI}=0.335-0.949$; overall coincidence rate $=87.8 \%)$. In terms of vaginal infiltration, pelvic examination and MRI showed moderate consistency $(K=0.434$; $95 \% \mathrm{CI}=0.119-0.733$; the OCR $=74.2 \%)$, and threedimensional TUI examination and MRI showed excellent consistency $(K=0.568,95 \% \mathrm{CI}=0.274-0.881$; the OCR $=81.9 \%)$. The accuracy of the backpropagation segmentation algorithm was $82 \%$. The results revealed that the three-dimensional TUI technology and MRI showed a good consistency in assessing parauterine invasion of cervical cancer. When the tumor size was evaluated, there was a clear correlation between three-dimensional TUI scans and MRI. Therefore, three-dimensional TUI technology showed relatively high application value for the local stage of cervical cancer.
\end{abstract}

\section{Introduction}

Cervical cancer is the fifth most common malignant tumor in women, and it is also one of the most common causes of cancer deaths in women, especially in underdeveloped countries [1-3]. The treatment of cervical cancer and after surgery mainly depends on the tumor stage $[4,5]$. However, most of such staging methods are based on the clinician's gynecological colposcopy and pelvic palpation. This is the independent judgment of the clinician and is affected by certain factors, such as inflammation and obesity, without any objective basis. There are inherent limitations in the assessment of tumor size, pericyst, pelvic tissue infiltration, and even the assessment of distant metastasis. Therefore, the purpose of segmentation cannot be achieved. Ultrasound is rarely used to assess the staging of cervical cancer [6]. 
However, the application of ultrasound imaging technology to the evaluation of cervical cancer staging has attracted special attention in recent years. Studies have reported that the sensitivity and specificity of ultrasound detection of parauterine invasion and prediction of advanced disease are $61 \%-82 \%$ and $87 \%-99 \%$, respectively [7]. Backpropagation algorithm, referred to as BP algorithm, is a learning algorithm suitable for multilayer neural networks, which is based on the gradient descent method. The inputoutput relationship of the BP network is essentially a mapping relationship, which is highly nonlinear. Its information processing ability comes from the multiple compounding of simple nonlinear functions, so it has strong function reproducibility. Ordinary two-dimensional ultrasound provides a dynamic evaluation of organ material anatomy during direct scanning. Compared with remaining static imaging techniques (such as magnetic resonance imaging (MRI) detection and computed tomography (CT) detection), its ability to dynamically observe anatomical structures is considered to be the theoretical benefit of ultrasound [8]. At present, three-dimensional ultrasound technology is widely used in clinical practice, and it can display the three-dimensional structure of tissues and organs [9]. Among them, tomographic ultrasound imaging (TUI) is a relatively new three-dimensional ultrasound imaging mode. This technology allows the evaluation of any desired observation plane of the anatomical area with a good impression and can perform multiple continuous tomographic scans of the anatomical area with an idea [10], such as CT and MRI. In addition, compared with conventional twodimensional ultrasound, TUI technology can generally accurately estimate the size and volume of the tumor and show the relationship between the lesion and the surrounding tissue more clearly [11]. It has been reported that the sensitivity and specificity of three-dimensional ultrasound for judging the peritumoral invasion of cervical cancer are $73 \%$ and $92 \%$, respectively [12].

Under the three-dimensional TUI of the backpropagation algorithm, this study was aimed to explore its application value in the local staging diagnosis of cervical cancer and provide a new method for clinical treatment of cervical cancer.

\section{Materials and Methods}

2.1. Research Objects. Thirty-five patients who were histologically diagnosed with cervical cancer after gynecological visits and cervical biopsy were included from January 2019 to November 2020. All patients underwent pelvic examination to determine the clinical stage, vaginal TUI, and MRI to determine the stage. The patients were $26-70$ years old, with an average of $51 \pm 10$ years old, and they all had a history of sexual behavior. There were 20 cases after menopause and 15 cases before menopause. This study had been approved by the Medical Ethics Committee of the hospital, and the family members of the patients included in the study had also signed the informed consent.

Inclusion criteria were given as follows: preoperative cervical biopsy confirmed cervical cancer and radical cervical resection was used as a reminder; transvaginal three- dimensional ultrasound tomography within 48 hours before surgery; and radical resection of cervical cancer, and the pathological diagnosis was IB1 $\sim$ Phase IIB. Exclusion criteria were defined as follows: preoperative radiotherapy and chemotherapy; no combined ultrasound or transvaginal ultrasound in our hospital within 48 hours before surgery; cervical cancer without ultrasound staging; and surgery confirmed lymph node metastasis.

2.2. Instruments. The color Doppler ultrasound diagnostic instrument was adopted, which was equipped with a 6-8 $\mathrm{MHz}$ vagina probe, TUI software, and $4 \mathrm{D}$ View 3D offline analysis software.

A double-blind method was adopted for routine ultrasound examination and TUI examination. Firstly, it should perform a routine transvaginal ultrasound examination. The patient was asked to empty the bladder, looked at the bladder stones, and applied with an appropriate amount of coupling agent to the probe. A sterile disposable sleeve was put into the probe. The acoustic window was between the probe and the cervix, and it was sent to the outer cervix. The power Doppler ultrasound was applied to evaluate the tumor vascular distribution. However, due to the difficulty of power Doppler imaging to determine the tumor boundary, this information is not used to evaluate the extent of local tumor invasion. No ultrasound examination of the pelvic lymph nodes was performed. After the 2D ultrasound inspection was completed, it could enter the $3 \mathrm{D}$ mode and adjust the parameters (scanning angle $118^{\circ}$, highest image quality, and highest sensitivity). The size of the sampling frame was adjusted according to the specific conditions of the lesion, and the cervix was placed as close as possible to the center of the image. It had to try to include the entire cervix, posterior bladder walls, and anterior rectum in the longitudinal plane, and activate the three-dimensional TUI system to obtain the three-dimensional images. The probe was rotated by $89^{\circ}$ to obtain a second $3 \mathrm{D}$ image on the cross section. Finally, the $3 \mathrm{D}$ images were saved. As with two-dimensional ultrasound, the lesion was measured in three planes, and then the largest diameter was used to determine the size of the lesion. The violation of the brain was evaluated via ultrasound tomography. When the outer cervix was intact and the boundary with the surrounding tissues was clear, it was felt that the area adjacent to the cancer had not been invaded (Figure 1 and 2). When the lesion interrupted the continuity of the outer cervix or damaged the surrounding tissues, it was suspected of invading the parauterine (Figure 3 ). The bladder and rectum were examined via the sagittal plane. When the tumor extended to the bladder or rectum and damaged the space between this part of the structure and the cervix, it was suspected of invading these structures.

\subsection{Image Segmentation Using Backpropagation Algorithm.} In the design and analysis of the backpropagation algorithm, the filter back-projection algorithm was easy to use in the ring probe. In the simulation process, because the found projection data was not a parallel beam, it cannot be used to use the filtered back-projection algorithm of the parallel 


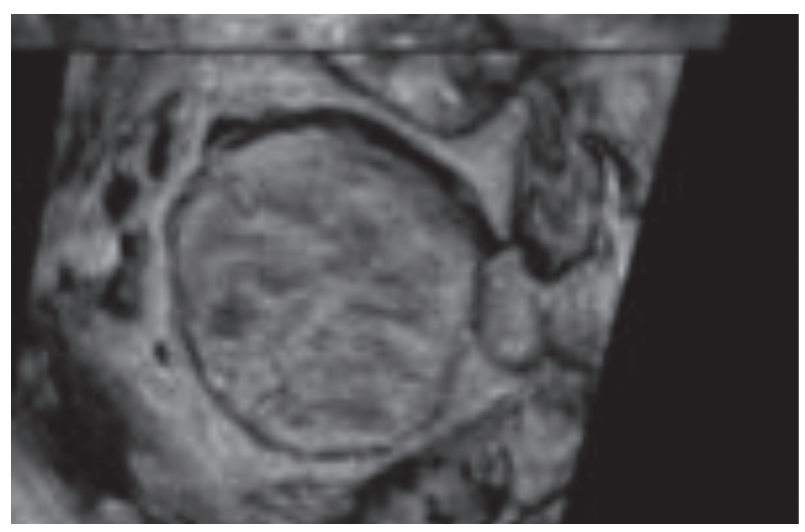

FIGURE 1: Image for tumor staging of IB.

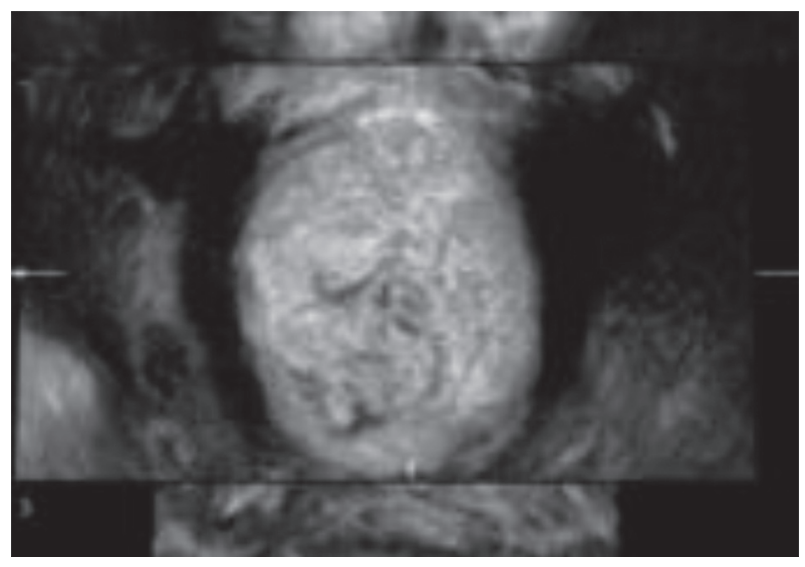

FIGURE 2: The image of the tumor stage of IIA2.

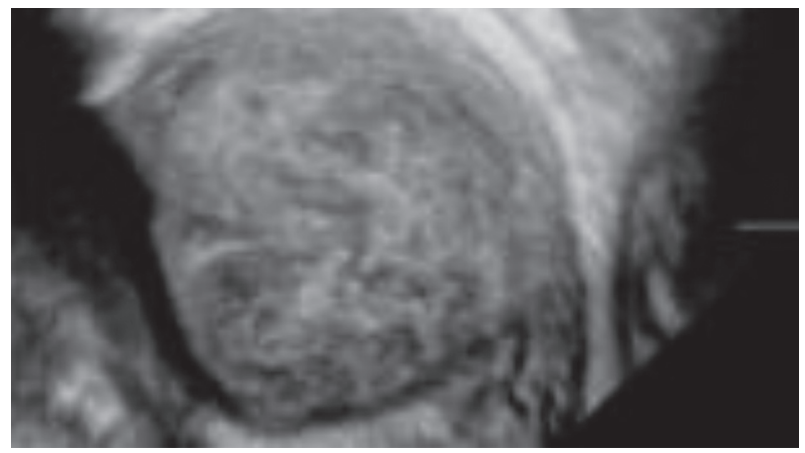

FIgURE 3: The image of tumor stage of IIIB.

beam. It can be applied after data rearrangement and interpolation. The completion of the algorithm had to first filter the projection data that were convolving with the filter.

If $n(i)$ was a filter function, then the below equation could be obtained. $j$ was the filtered projection, $i$ was the variable, $\theta$ referred to the angle, $D$ represented the projection, and $p$ was the distance between parallel projection rays.

$$
j(i, \theta)=\int_{-\infty}^{\infty} \mathrm{D}\left(i^{\prime}, \theta\right) n\left(i-i^{\prime}\right) p i^{\prime} .
$$

In theory, the frequency domain response of $n(i)$ is required to be $N(\mu)=|\mu|$. According to the Paley-Wiener rule, this kind of ideal filter is unlikely. It was assumed that the bandwidth of the Fourier transform of the projection is limited, and the energy outside the frequency $(-\Phi, \Phi)$ was 0 . To ensure nonaliasing sampling, the projection bandwidth should meet the Nyquist sampling criterion:

$$
\Phi=\frac{1}{2} \sigma .
$$

In the above equation, $\sigma$ was the projection sampling interval, so the ramp function $|\mu|$ was actually multiplied by the window function $r(\mu)$.

$$
N(\mu)=|\mu| r(\mu) .
$$

Of that,

$$
r(\mu)=\left\{\begin{array}{l}
1.2,|\mu|<\Phi \\
0,|\mu| \geq \Phi
\end{array}\right.
$$

The expression of the impulse response in the spatial domain of the filter was as follows:

$$
\begin{aligned}
n(i)= & \int_{-\Phi}^{\Phi}|\mu| \int e^{g 2 \pi \mu i} p \mu=\frac{1}{3 \sigma}\left(\frac{\cos 3 \pi \Phi i}{2 \pi \phi t}\right) \\
& -\frac{1}{6 \sigma^{3}}\left(\frac{\cos \pi \Phi i}{\pi \Phi i}\right)^{23} .
\end{aligned}
$$

In the discrete completion of the filter projection, the filter value at an integer multiple of $\sigma$ was thought of, and the impulse response of the filter can be obtained by substituting $i=h \sigma$ into (5):

$$
n(h \sigma)= \begin{cases}\frac{1}{5 \sigma^{3}}, & n=0 \\ 0, & h \text { is an even number, } \\ -\frac{1}{(h \pi \sigma)^{3}}, & h \text { is an odd number. }\end{cases}
$$

The structure of the back-propagation algorithm network data analysis model is shown in Figure 4.

2.4. Pelvic Examination. All patients were clinically staged according to the standards of the Federation Internationale of Gynecologyie and Obstetrigue (FIGO). All patients were examined by a gynecologist. The binomial and triple examination was adopted to evaluate the size of the tumor and whether it involved the vagina, uterus, bladder, and rectum, and then the endoscopy was performed.

2.5. MRI Examination. All cervical cancer patients were examined by an MRI machine. The conventional MRI scan was performed firstly, and then the pelvic phase anterior ring was performed to scan the T1 and T2-weighted phase scans of the cervical spine, sagittal, and coronal planes. The layer thickness was $6 \mathrm{~mm}$, the interval was 1.1 or $1.6 \mathrm{~mm}$ according to the tumor size, and the stroma was $510 \times 255$. 


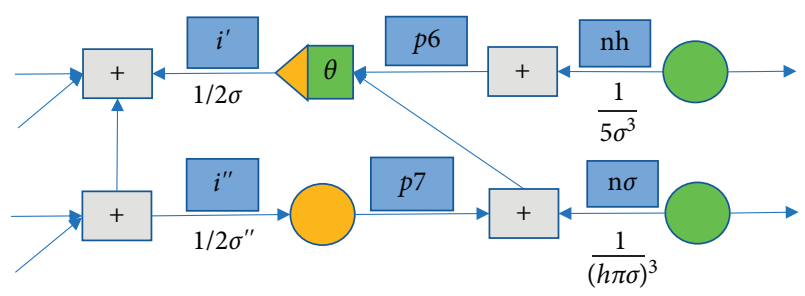

FIGURE 4: The network data analysis model of the backpropagation algorithm.

The tumor showed medium, high, and mixed signals in the T2-weighted phase. The MRI doctor reviewed the X-ray film and evaluated its stage (Figure 5).

2.6. Observation Indicators. In the image segmentation effect, the image segmentation index was used. Under normal circumstances, the prediction result obtained by the segmentation algorithm should be compared with the actual result of the label. It should measure and observe the tumor size, parauterine invasion, and vaginal invasion under three inspections, namely, 3D tomography, MRI, and pelvic examination. The reliable conclusions could be drawn under careful comparison.

2.7. Statistical Analysis. The SPSS 21.0 software was adopted for statistical analysis. The measurement data were expressed as the mean \pm standard deviation and difference. Categorical variables were expressed in absolute numbers and percentages. The chi-square test was used for normally distributed data, and the rank-sum test was used for nonnormally distributed data. The consistency test (Kappa coefficient) was used to determine the consistency of three-dimensional tomography, pelvic examination, and MRI in assessing the paracerebral, vaginal, and aspects. If the $\mathrm{K}$ value was less than 0.19 , the consistency was considered poor; if it was 0.20 to 0.39 , the consistency was considered basically consistent; if it was 0.40 to 0.59 , the consistency was considered to be medium; if the value was 0.60 to 0.79 , good consistency was determined; and if the value was 0.80 to 1.00 , it was considered very good consistency. Pearson coefficient $(R)$ was used to evaluate the correlation of three-dimensional tomography, pelvic examination, and MRI to evaluate tumor size. $P<0.05$ was considered statistically significant.

\section{Results}

3.1. Basic Information of Patients. A total of 35 cases were collected in this study. Among the 35 cases of cervical cancer, 29 cases $(83 \%)$ of squamous cell carcinoma and 6 cases $(17 \%)$ of adenocarcinoma were pathologically diagnosed. According to the classification of the FIOG, the tumor staging was as follows: IA stage: $n=1$ (3\%); IB 1 stage: $n=9(25.3 \%)$; IB 2 stage: $n=10$ (27.5\%); IIA 1 stage: $n=4$ (15.6\%); IIA 2 stage: $n=2$ (3.1\%); IIB stage: $n=3(12.8 \%)$; IIIB stage: $n=4(15.6 \%)$; and IVA stage: $n=1(3.1 \%) .11$ patients (all in IA and IB 1 stages) made surgery the main treatment modality, 24 (all IB 2-IVB stages) made

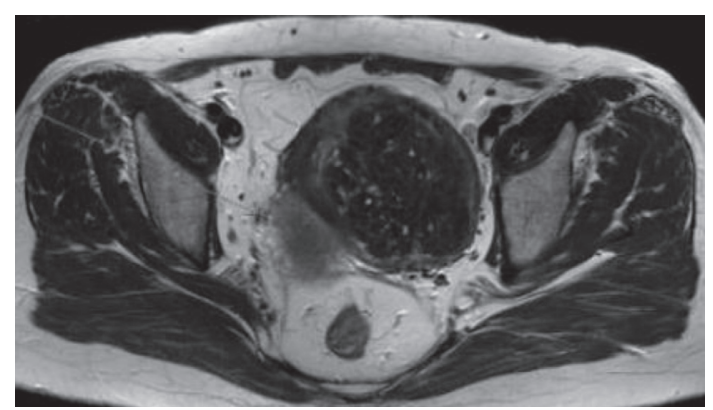

FIGURE 5: MRI images of patients with cervical cancer.

radiotherapy the main treatment modality, and chemotherapy helped the treatment.

\subsection{Evaluation of Three-Dimensional TUISegmentation Effect} Based on Backpropagation Algorithm. In the process of using the algorithm, there would be some errors, such as the inherent error of the algorithm, the grid processing of the imaging area, the accuracy of the data completion process, and the error of the interpolation operation. By optimizing the system structure, some errors can be reduced. Facing the circular array, if you want to enhance the accuracy of the system, it can use methods such as increasing the number of probes and increasing the imaging angle. Facing the triangular array, because its imaging angle was fixed, the way to improve the accuracy of the system was to increase the number of probes. The increase in the number of probes and imaging angles inevitably led to an increase in imaging time and imaging costs. Therefore, it was necessary to analyze the consideration of the number of probes and the imaging angle on the imaging quality and find a convenient imaging method. The main technical indicators were as follows: the automatic segmentation accuracy was greater than $82 \%$, the reconstruction accuracy of the multidimensional cervix can be observed at any angle, and the reconstruction accuracy was not lower than the previous input ultrasound resolution. The calculation error was less than 9\%, the average accuracy had reached $74.7 \%$, and the memory footprint was less. Memory is another meaningful factor of the segmentation method. Although the limit is looser than the execution time and the memory can be obtained more flexibly, it is still a constraint factor. In some cases, such as on-chip operating systems and robotic platforms, its memory resources are not abundant compared to high-performance servers. Even if the high-end graphics processing of the deep network can be accelerated, the memory resources are relatively limited. From this point of view, it is extremely valuable to record the extreme value and average value of the memory occupied by the system in the running state under the same running time. The execution time is shortened. Figure 6 shows the ultrasound image of the cervix and the result of the target region segmentation algorithm in the ultrasound image.

3.3. Comparison of Inspection Results. The results of threedimensional tomography, MRI, and pelvic examination were compared, as shown in Figures 7-9. The results of TUI, 


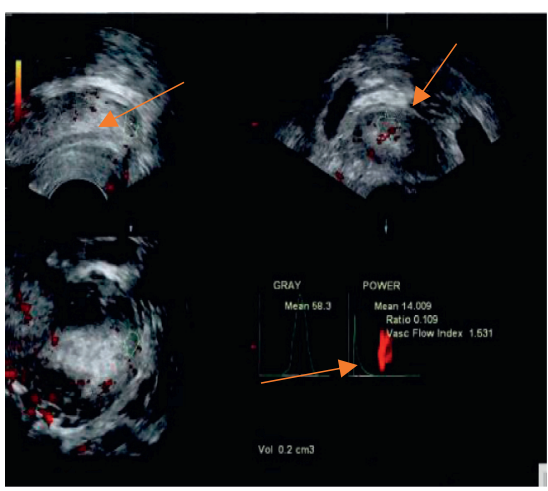

(a)

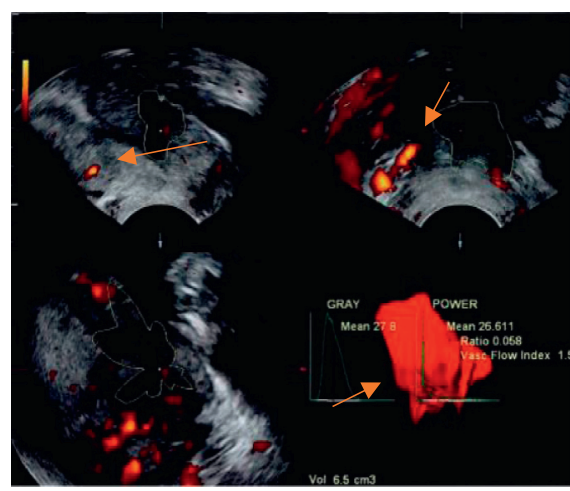

(b)

FIgURE 6: The ultrasound image of the cervix (a) and the result of the algorithm segmentation of the target region in the ultrasound image (b).

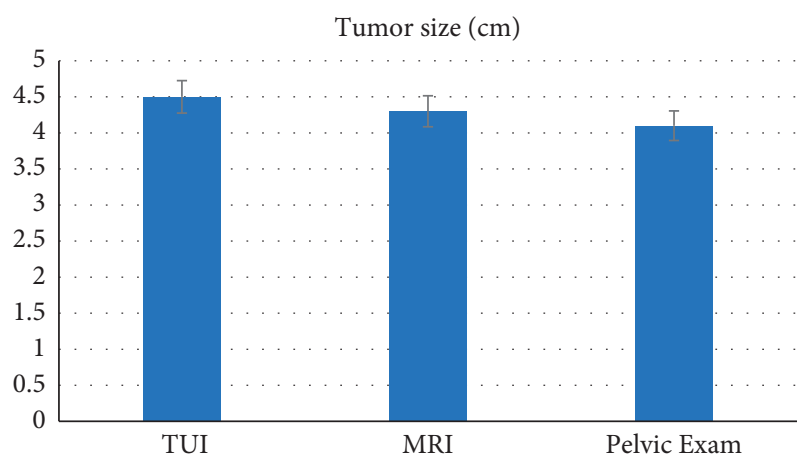

Figure 7: Comparison of tumor size $(\mathrm{cm})$.

MRI, and pelvic examination were not statistically different $(P>0.05)$, so they were comparable.

Comparison of tumor size measurements showed that obvious correlation could be found between MRI and threedimensional TUI $(r=0.842 ; P<0.001), \mathrm{MRI}$, and clinical examination $(r=0.654 ; P<0.001)$ to the assessment of tumor size. In terms of parauterine invasion, there was a moderate consistency between pelvic examination and MRI $(K=0.413$; $95 \%$ confidential interval $(C I)=0.085-0.762$; the overall coincidence rate $(\mathrm{OCR})=78.0 \%)$, there was good consistency between three-dimensional TUI and MRI ( $K=0.655,95 \% \mathrm{CI}=0.335-0.949$; OCR $=87.8 \%)$. In terms of vaginal infiltration, pelvic examination and MRI showed moderate consistency $(K=0.434 ; 95 \% \mathrm{CI}=0.119-0.733$; the OCR $=74.2 \%$ ), and three-dimensional TUI examination and MRI showed excellent consistency $(K=0.568, \quad 95 \%$ $\mathrm{CI}=0.274-0.881$; the $\mathrm{OCR}=81.9 \%)$. The involvement of the bladder and rectum was not involved because the number of cases was somewhat small.

\section{Discussion}

The current clinical staging is still the standard for cervical cancer staging, and clinical evaluation is still very important [13]. Imaging or pathological evidence is included in the latest FIGO stage of cervical cancer in 2019, and all stages can be supplemented by imaging and pathology [14]. The result

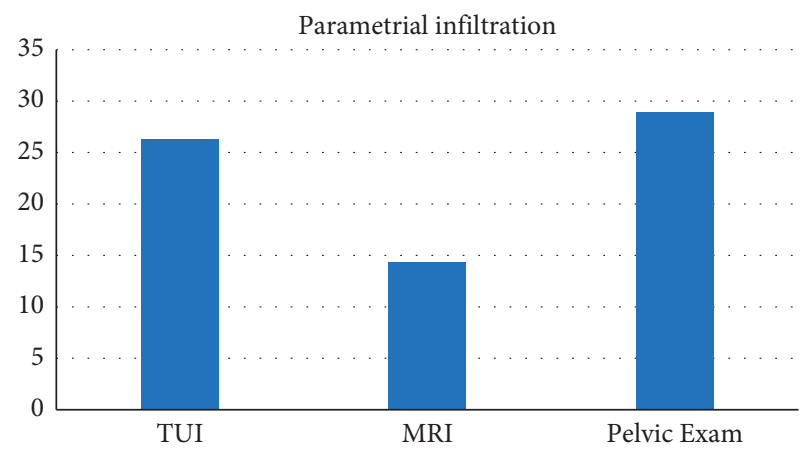

Figure 8: Comparison of the penetration examination results of the tissues around the uterus.

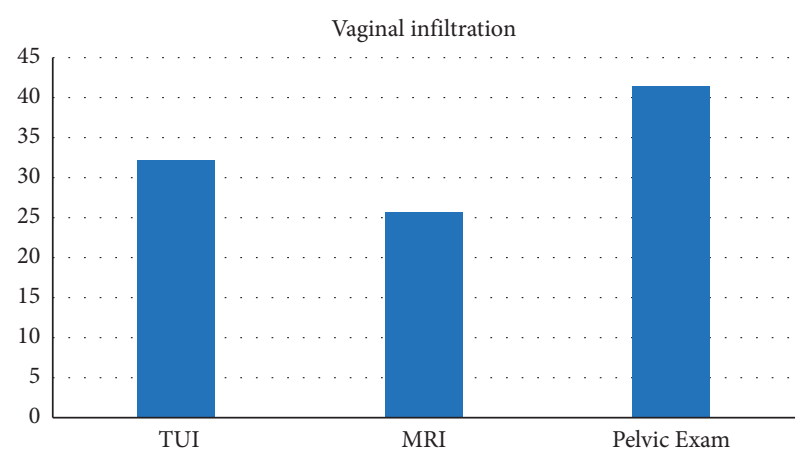

Figure 9: Comparison of vaginal penetration test results.

of the imaging examination is to find the most suitable treatment method and prevent the combined treatment of surgery and radiotherapy from deepening the bad response. MRI is currently considered to be the primary imaging technique to assess the degree of local invasion of cervical cancer $[15,16]$. The results of this study were in line with our initial expectations. Backpropagation algorithm technology can evaluate any required plane of the anatomical area of interest and can perform multiple continuous plane tomography of the anatomical area of interest, which is of great significance in the diagnosis of cervical cancer. This study evaluated the consistency of TUI examination and MRI 
examination in cervical cancer staging assessment and explored the possibility and potential application value of TUI examination technology in cervical cancer staging assessment [17]. The results revealed that TUI and MRI showed good consistency and correlation in assessing parauterine and vaginal invasion and judging tumor size. As mentioned above, MRI is indeed an excellent imaging technique for assessing the local invasion of cervical cancer and a collaborative tool for clinical staging [18]. For the evaluation of subparauterine invasion, it had obtained the consistency of TUI and MRI, and the results showed that the consistency of the two inspection methods was better. Therefore, it can be concluded that TUI ultrasound has a good consistency with the current standard technology (MRI) and can be used for the assessment of the local lesions of cervical cancer [19]. This was consistent with the findings of Sara Arribas et al. [20]. The advantages of TUI technology were as follows: Firstly, the transvaginal probe used by TUI has a high resolution, shows a short distance to the lesion, and is flexible and convenient to operate. Secondly, TUI not only uses volume imaging to enhance contrast, and it can also strongly evaluate soft tissues. In addition, the image can be expressed in the form of continuous failure, and the desired image can be obtained by adjusting the thickness and number of layers in real time, thereby observing the tumor from multiple directions. Thirdly, TUI can perform multidirectional and multidirectional detection of areas of interest according to needs. The inspection time is short and the operation is convenient, which is exactly what MRI cannot achieve. Fourthly, TUI can obtain the sagittal, coronal, and transverse planes of the tumor and measure the maximum diameter line of the tumor on three planes, so as to more accurately observe the tumor size, especially the endogenous and irregular lesions [21]. Fifthly, patients with metal in their bodies cannot undergo MRI, and MRI is relatively expensive [22]. However, TUI can be suitable for all patients, and the cost is significantly lower than that of MRI.

In this study, there was no comparison between the results of ordinary two-dimensional transvaginal ultrasound and three-dimensional TUI. Since satisfactory three-dimensional TUI images are rebuilt from two-dimensional images, these two methods are inseparable and can complement each other. Some studies have shown that three-dimensional ultrasound is more accurate in evaluating tumor volume than two-dimensional ultrasound. However, there were some limitations for this study. Firstly, all ultrasound examinations were performed by the same doctor, and there was no assessment of differences between operators. Secondly, the number of cases in this study was relatively small, especially the number of late bladders and rectal infiltration. The imaging features of early infiltration of some adjacent tissues needed to be observed and summarized on a large number of samples for early detection. In addition, TUI itself has certain limitations. TUI imaging technology is based on two-dimensional ultrasound imaging, so the operation and proficiency of the surgeon will affect the quality of image acquisition. In summary, the three-dimensional TUI technology can accurately assess the local infiltration of cervical cancer lesions and provide more objective imaging data for the clinic, so it is an examination technology that can be further researched and promoted.

\section{Conclusion}

Three-dimensional TUI technology and MRI had good consistency in evaluating cervical cancer parauterine invasion. When the tumor size was evaluated, there was a clear correlation between three-dimensional TUI and MRI. The three-dimensional TUI technology showed good application value for local staging of cervical cancer. There were also many shortcomings and deficiencies in the study. In this study, the sample size was too small, and more people should be included in the experiment. The clinical trials should not be conducted in a single area or a small area in a multicenter, large-sample hospital.

\section{Data Availability}

The data used to support the findings of this study are available from the corresponding author upon request.

\section{Conflicts of Interest}

The authors declare that there are no conflicts of interest.

\section{Authors' Contributions}

Chenjie Li and Ziwen Zhang contributed equally to this work.

\section{References}

[1] S. Wei, P. Dai, and Z. Wang, "Cervical cancer detection and diagnosis based on saliency single shot MultiBox detector in ultrasonic elastography," Journal of Medical Systems, vol. 43, no. 8, p. 250, 2019.

[2] F. Fukunari, K. Okamura, R. Zeze, T. Kagawa, K. Hashimoto, and K. Yuasa, "Cervical lymph nodes with or without metastases from oral squamous carcinoma: a correlation of MRI findings and histopathologic architecture," Oral Surgery, Oral Medicine, Oral Pathology, Oral Radiology \& Endodontics, vol. 109, no. 6, pp. 890-899, 2010.

[3] Y. Zhang, Y. Yan, and Y. Yang, "Study on value of ultrasonic elastography in diagnosis of clinical staging of cervical cancer and efficacy evaluation of radiotherapy," Oncology Letters, vol. 17, no. 6, pp. 4901-4906, 2019.

[4] J. Zhou, S.-Y. Zhu, R.-C. Liu, F. Luo, and D.-X. Shu, "Vascularity index of laryngeal cancer derived from 3-D ultrasound: a predicting factor for the in vivo assessment of cervical lymph node status," Ultrasound in Medicine and Biology, vol. 35, no. 10, pp. 1596-1600, 2009.

[5] Y. Xu, T. Ru, L. Zhu et al., "Ultrasonic histogram assessment of early response to concurrent chemo-radiotherapy in patients with locally advanced cervical cancer: a feasibility study," Clinical Imaging, vol. 49, pp. 144-149, 2018.

[6] L. Xu, Q. Zhang, C. Li, F. Hua, and X. Liu, "Ultrasound microbubble-mediated microRNA-505 regulates cervical cancer cell growth via AKT2," Analytical Cellular Pathology, vol. 2020, Article ID 3731953, 2020. 
[7] O. A. Bakay and T. S. Golovko, "Use of elastography for cervical cancer diagnostics," Experimental Oncology, vol. 37, no. 2, pp. 139-145, 2015.

[8] K. Zhang, H. Wang, Z. Wang et al., "Intensity-modulated radiation therapy (IMRT)-based concurrent chemoradiotherapy (CCRT) with Endostar in patients with pelvic locoregional recurrence of cervical cancer," Medicine, vol. 99, no. 49, Article ID e21966, 2020.

[9] K. R. Carr, Y. J. Ioffe, M. Filippova, P. Duerksen-Hughes, and P. J. Chan, "Combined ultrasound-curcumin treatment of human cervical cancer cells," European Journal of Obstetrics \& Gynecology and Reproductive Biology, vol. 193, pp. 96-101, 2015.

[10] X.-S. Han, C.-P. Ning, L.-T. Sun, X.-Y. Li, Y.-Q. Peng, and M.-Z. Dang, "Three-dimensional transvaginal tomographic ultrasound imaging for cervical cancer staging," Ultrasound in Medicine and Biology, vol. 41, no. 9, pp. 2303-2309, 2015.

[11] M. Akazawa, T. Saito, M. Okadome, and K. Ariyoshi, "Comparison of electrosurgical devices for cervical conization: novel monopolar scalpel (VIO) versus ultrasonic scalpel," Journal of Lower Genital Tract Disease, vol. 23, no. 1, pp. 43-47, 2019.

[12] Q. Zhu, Z. Shao, X. Zhang, and D. Xu, "Correlation between ultrasonic features of medullary thyroid carcinoma and cervical lymph node metastasis," Ultrasound in Medicine and Biology, vol. 47, no. 4, pp. 967-972, 2021.

[13] C. Mou, F. J. Lü, B. Yu, and Z. B. Xiao, "[The value of MRI in early cervical cancer staging with vaginal filling of ultrasound gel]," Sichuan Da Xue Xue Bao Yi Xue Ban, vol. 52, no. 3, pp. 523-527, 2021.

[14] H. C. Kim, D. Y. Yoon, S. K. Chang et al., "Small atypical cervical nodes detected on sonography in patients with squamous cell carcinoma of the head and neck," Journal of Ultrasound in Medicine, vol. 29, no. 4, pp. 531-537, 2010.

[15] F. Ahmadi, M. Ghasemi-Kasman, S. Ghasemi et al., "Induction of apoptosis in HeLa cancer cells by an ultrasonicmediated synthesis of curcumin-loaded chitosan-alginate-STPP nanoparticles," International Journal of Nanomedicine, vol. 12, pp. 8545-8556, 2017.

[16] S. Kar and D. D. Majumder, "A novel approach of mathematical theory of shape and neuro-fuzzy based diagnostic analysis of cervical cancer," Pathology and Oncology Research, vol. 25, no. 2, pp. 777-790, 2019.

[17] B. Hohlweg-Majert, M. C. Metzger, P. J. Voss, F. Hölzle, K.-D. Wolff, and D. Schulze, "Preoperative cervical lymph node size evaluation in patients with malignant head/neck tumors: comparison between ultrasound and computer tomography," Journal of Cancer Research and Clinical Oncology, vol. 135, no. 6, pp. 753-759, 2009.

[18] Z. Wu, X. Peng, J. Li, Y. Zhang, and L. Hu, "Constitutive activation of nuclear factor $\kappa \mathrm{B}$ contributes to cystic fibrosis transmembrane conductance regulator expression and promotes human cervical cancer progression and poor prognosis," International Journal of Gynecological Cancer, vol. 23, no. 5, pp. 906-915, 2013.

[19] X. Peng, Z. Wu, L. Yu et al., "Overexpression of cystic fibrosis transmembrane conductance regulator (CFTR) is associated with human cervical cancer malignancy, progression and prognosis," Gynecologic Oncology, vol. 125, no. 2, pp. 470-476, 2012.

[20] S. Arribas, J. L. Alcázar, M. Arraiza, A. Benito, J. A. Minguez, and M. Jurado, "Three-dimensional transvaginal sonography and magnetic resonance imaging for local staging of cervical cancer," Journal of Ultrasound in Medicine, vol. 35, no. 5, pp. 867-873, 2016.

[21] H. Zhang, C. Chen, R. Gao et al., "Rapid identification of cervical adenocarcinoma and cervical squamous cell carcinoma tissue based on Raman spectroscopy combined with multiple machine learning algorithms," Photodiagnosis and Photodynamic Therapy, vol. 33, Article ID 102104, 2021.

[22] M. Ekpenyong, A. Asitok, S. Antai, B. Ekpo, R. Antigha, and N. Ogarekpe, "Statistical and artificial neural network approaches to modeling and optimization of fermentation conditions for production of a surface/bioactive glyco-lipopeptide," International Journal of Peptide Research and Therapeutics, vol. 27, no. 1, pp. 475-495, 2020. 